\title{
Utilization of Long Lasting Insecticidal Nets Among Household in Malarious Areas of Raya Alamata District, Tigray, Ethiopia
}

\author{
Girmay Desta Araya ${ }^{1}$, Hailemariam Lemma Reda ${ }^{2}$, Azeb Gebresilassie Tesema ${ }^{3,}$ * \\ ${ }^{1}$ Tigray Regional Health Bureau, Mekelle, Tigray, Ethiopia \\ ${ }^{2}$ PATH/ Malaria Control and Evaluation Partnership in Africa (MACEPA)-Ethiopia \\ ${ }^{3}$ Mekelle University, College of Health Science, Department of Public Health, Mekelle, Ethiopia
}

Email address:

girma_de@yahoo.com (G. D. Araya), hailelm@gmail.com (H. L. Reda), azeb18@gmail.com (A. G. Tesema)

\section{To cite this article:}

Girmay Desta Araya, Hailemariam Lemma Reda, Azeb Gebresilassie Tesema. Utilization of Long Lasting Insecticidal Nets Among Household in Malarious Areas of Raya Alamata District, Tigray, Ethiopia. Science Journal of Public Health.

Vol. 3, No. 2, 2015, pp. 216-221. doi: 10.11648/j.sjph.20150302.19

\begin{abstract}
Background: Malaria in Ethiopia is one of the most important public health problems, with more than threequarters of the landmass of the country and an estimated $68 \%$ of the total population is considered at risk of malaria infections. In the fight against this deadly disease, the country has deployed a massive amount of long lasting Insecticidal treated nets. Though this tremendous effort is outstanding, the distribution alone does not ensure the protection of the people from malaria. Thus, this study tried to assess the utilization of long lasting insecticidal nets and its associated factors among household in malarious areas of Raya Alamata District, Tigray, Ethiopia. Methods: Community based cross sectional study was used among 649 households from April to May, 2013. All households in malarious villages in Raya Alamata district with long lasting Insecticidal nets in their home were used and multistage sampling technique was used to select the study subjects. For day time face to face interview, structured questionnaire was used. Additionally, an observation checklist was used for early morning survey of the actual utilization. Descriptive statistics and binary logistic regression analysis were performed. Results: Among all households $445(68.6 \%)$ of them were utilize the long lasting Insecticidal treated nets. The proportion of under 5 children and pregnant women who slept under the net during early morning survey was $71.64 \%$ and $69 \%$ respectively. The number of net in the households [AOR $(95 \% \mathrm{CI})=0.085(0.26-0.281)$ ], household's marital status [AOR $(95 \% \mathrm{CI})=0.431(0.186-0.997)$ ] and occupation of the household head [AOR $(95 \% \mathrm{CI})=0.297(0.113-0.781)]$ were the significant predicators for utilization of long lasting Insecticidal treated nets. Conclusions: Utilization of long lasting insecticidal nets in this study is low and there is a considerable discrepancy between the distribution and utilization of the net. Availability of number of nets among the households, occupational status the head of households and marital status of the household are the predictor variables for household's utilization of the net.
\end{abstract}

Keywords: Long Lasting Insecticidal Treated Nets, Utilization

\section{Introduction}

Malaria is the most important parasitic infection in endemic areas and life treating diseases, transmitted from one human to another by the bite of infected Anopheles mosquitoes (1). Despite being a preventable and treatable disease, globally, malaria remains to be a major problem causing an unacceptable toll on the health and economic welfare of world's poorest communities. There were an estimated 198 million malaria cases and 584,000 deaths (uncertainty range 367 000-755 000) malaria deaths in 2013, of which $90 \%$ were in the African Region. Approximately $78 \%$ of malaria deaths globally were of children under 5 years of age (2).

Malaria is highly intense in sub Saharan African (SSA) countries, accounting for $25-35 \%$ of all outpatient visits, 20 $45 \%$ of hospital admissions and $15-35 \%$ of hospital deaths, imposing a great burden on already existing health-care systems. Worldwide, about $60 \%$ of the cases of malaria, $75 \%$ of global falciparum malaria cases and more than $80 \%$ of malaria deaths occur in this region(3).

Ethiopia is among the top five main contributing countries 
to the overall African malaria burden. Despite the long history of malaria eradication and control since the 1950s, malaria still a major public health problem in the country and main causes of hospitalization and death in all corners of the country (4). Malaria in Ethiopia is seasonal, predominantly unstable and focal, depending largely on rain fall and altitude. Two transmission seasons are known the first transmission period is from September to December and the short transmission period also April to May. The unstable nature of malaria makes the population non-immune and prone to focal and cyclical epidemics (5).

Under the key Roll Back Malaria program one of the global strategic interventions is to fight against malaria by is the protection of people at risk from malaria using locally appropriate vector control methods such as Insecticide Treated Nets (ITNs) (6). Long lasting insecticidal nets (LLINs) have become important tool that provide a simple and effective means of preventing malaria in highly endemic areas (7).

The effectiveness of LLINs intervention depends on high coverage and effective utilization to boost ITNs distribution through the public and private sectors, since 2002 the government has removed waived the tax on LLINs (8). The Government of Ethiopia is highly committed and realizing the LLINs for prevention of malaria transmission and LLINs should be provided to households in malaria endemic areas i.e. that are below 2,000m above the sea level (9). Different study suggested that utilization of LLINs is still unacceptably low that, the proportion of households owning at least one LLIN in sub-Saharan is $50 \%$ in 2011 . Worse still; malaria continues to be the leading cause of child mortality and morbidity $(2,7)$.

Though, substantial increase has been reported regarding household LLINs ownership in Ethiopia, barriers ranging from house structure to user behavior are becoming obstacles for optimal use. Lacks of knowledge regarding priority groups to sleep under LLINs in the household and inappropriate usage are frequently reported to hamper effective utilization of LLINs in different parts of Ethiopia (10). The situation may have a similar scenario in the study area, Raya Alamata district, Southern Tigray Ethiopia. In addition to the above barriers, experience from the field has shown that the communities in the study area were seen using LLINs for others purposes like, window curtain and hair covering than for protection of mosquito biting. Therefore, this study was aiming to assess the utilization and factors associated with utilization of LLINs among household of Malarias area of Raya Alamata District, Southern Tigray, Ethiopia.

\section{Methods and Materials}

\subsection{Study Design and Setting}

Community based cross sectional study was used from April to May, 2013. The study was conducted in the malarious district of Raya-Alamata, Southern Tigray region,
Ethiopia. According to the 2007 nation census the projected total population of the district for the year 2011 was 97,101 . As per to the district health office annual report, out of the total population $73 \%$ people are residing at area risk for malaria and according to the health facilities report malaria is among the leading causes of illness and death constituting $40.4 \%$ of all out patients cases (8). Malaria transmission in the district is bimodal and unstable (seasonal). The major transmission period is between September to December after the main rainy season and the minor is between April to May following a short rainy season (5 .8).

\subsection{Study Population and Sampling Techniques}

The study subjects were all households in malarious villages in Raya Alamata district who had at least one LLINs. Sample size was determined with the assumption of single population proportion considering proportion of $73 \%$ LLINs utilization in malarious villages of Arbaminch, Ethiopia (7), considering 5\% margin of error, $95 \%$ Confidence Interval (CI) and $10 \%$ non-response rate with a design effect of two to allow clustering effect. The final sample size was computed to be 667 . Multistage sampling method was used where five were selected from the total 10 malaria endemic village. A systematic random sampling method with proportional to size was used to select the study households.

\subsection{Data Collection Technique and Data Quality Control}

Structured face to face interview questionnaire which includes socio demographic and other characteristics that can measure factors associated with utilization of LLINs. An observation checklist was used for early morning survey of LLINs actual utilization was employed. Selected households were visited twice. Firstly, in early morning in order to observe the actual practice of LLINs use before the families leave their sleeping place. Secondly during the day time households were visited again for the interview. The respondents were the head of the house hold either wife or husband.

\subsection{Data Management and Analysis}

Data were checked for completeness and consistency entered and analyzed using SPSS version 16. Frequencies and percentages were used to describe the socio demographic characteristics and other variables. Logistic regression analysis was done to compute crude odds ratio (COR) and adjusted odds ratio (AOR) with $95 \%$ confidence intervals. The major factors influencing LLINs utilization were computed and interpreted at $\mathrm{p}<0.05$.

\subsection{Operational Definitions}

LLINs utilization- The use of standardized properly hanged (mounted) over the bed or the sleeping area and less than 5 years of age child, pregnant women and other population group sleeping under the mosquito net during the early morning of observation day. 


\subsection{Ethical Consideration}

Ethical clearance was obtained from Mekelle University, College of Health Sciences, Research and Community service office. Official permission letter was secured from Tigray Regional Health Bureau and Raya Alamata district health office.

Prior to interview and inspection of household survey, data collectors requested for the respondents' consent. Respondents were informed about the confidentiality of the information they provided. Data collectors were health professionals and created a trusting environment by respecting participants' privacy that can encourage them to be as honest as possible during the course of the survey. Participation in the survey was voluntary and they could choose not to participate, but was told that their views will be important to strengthen the program. When the data collectors found problems with incorrect use of nets, they educated the study participants about the correct use and maintenance upon completion of the survey. Clinically ill patients found during the survey advised to consult the nearby health facility to get health service.

\section{Results}

\subsection{Socio-Demographic Characteristics of Study Participants}

Of 667 households in malarious villages in Raya Alamata district with LLINs in their home were selected for the study, 649 households responded for the questionnaire, yielding a response rate $97.3 \%$. Out of these $467(72 \%)$ of the respondents were female and in terms of sex composition of house hold heads majority 538 (82.9\%) of them were males. The median age of the respondents was 35 years. Educationally, $414(63.8 \%)$ of the respondents did not able to read and write. Almost all $612(94.3 \%)$ of the respondents were orthodox Christian by religion. Regarding marital status of the respondents 552(85.1\%) was married. The occupation of the head of household, 586(90.3\%) of them were farmers. The mean family sizes of the household were $5.14+1.32$ SD Majority $460(70.9 \%)$ of them had only one sleeping room for the family (Table 1).

Table 1. Socio-demographic characteristics of respondents of Raya-Alamata, Tigray region, Ethiopia, $2013(n=649)$.

\begin{tabular}{ll}
\hline Variables category & $\mathbf{n}(\mathbf{\%})$ \\
\hline Sex of respondents & \\
Female & $467(72)$ \\
Male & $182(28)$ \\
Age of respondents & \\
$18-24$ years & $37(5.7)$ \\
$25-30$ years & $226(34.8)$ \\
$31-39$ years & $123(19)$ \\
$>=40$ years & $263(40.5)$ \\
Sex of the head of Household & \\
Male & $538(82.9)$ \\
Female & $111(17.1)$ \\
Educational status of the head of Household & \\
Illiterate & $414(63.8)$ \\
\hline
\end{tabular}

\begin{tabular}{ll}
\hline Variables category & n (\%) \\
\hline Primary & $137(21.1)$ \\
Read and write & $72(11.1)$ \\
Secondary and above & $26(4)$ \\
Religion of the head of household & \\
Orthodox & $612(94.3)$ \\
Muslim & $37(3.7)$ \\
Marital status head of the household & \\
Married & $552(85.1)$ \\
Divorced & $56(8.6)$ \\
Single & $15(2.3)$ \\
Widowed & $26(4)$ \\
Occupational status of the household & \\
Farmer & $586(90.3)$ \\
Day labor & $24(3.7)$ \\
Merchant & $23(3.5)$ \\
Gov / private Employed & $16(2.5)$ \\
Number of sleeping rooms & \\
One & $460(70.9)$ \\
Two & $162(25)$ \\
Three & $27(4.2)$ \\
Mean Family size = 5.14 and SD=1.323 & \\
\hline
\end{tabular}

\subsection{Long Lasting Insecticidal Nets (LLINs) Utilization Among Households}

Table 2. LLINs utilization by households in Raya-Alamata district, Tigray, Ethiopia, $2013(n=649)$.

\begin{tabular}{ll}
\hline Variables Category & $\mathbf{n}(\mathbf{\%})$ \\
\hline HHs who hanged LLINs & \\
Yes & $445(68.6)$ \\
No & $204(31.4)$ \\
Number of LLINs hanged among the HHs & \\
One & $335(51.6)$ \\
Two & $262(40.4)$ \\
Three & $52(0.8)$ \\
Total number of LLINs used & \\
Yes & $678(66.8)$ \\
No & $337(33.3)$ \\
LLINs tucked under the mattress & \\
Yes & $232(35.7)$ \\
No & $417(64.3)$ \\
LLINs has tears or holes & \\
Yes & $65(10)$ \\
No & $584(90)$ \\
Any repaired holes on the LLINs & \\
Yes & $29(4.5)$ \\
No & $620(95.5)$ \\
Utilization of LLINs during early morning survey & \\
Adult and other population group & $1,152(68)$ \\
Under five children & $326(71.64)$ \\
Pregnant mother & $83(69)$ \\
Reasons for LLINs not hanged & \\
Forgotten & $95(46.6)$ \\
No insects & $10(4.9)$ \\
They have tears and holes & $19(9.3)$ \\
The LLINs is too hot & $32(15.7)$ \\
No important if a chemical is sprayed & $11(5.4)$ \\
LLINS have chemicals & $20(9.8)$ \\
\hline & $17(8.3)$ \\
\hline
\end{tabular}

Among all households $445(68.6 \%)$ of them were hanged or utilize the LLINs and the rest 204(31.4\%) of them were not hanged the net. The net was given based on the size of the family and some of the household had more than one net. Accordingly, among all the households $314(41.2 \%)$ of them 
had more than one LLINs. In terms of number from the total available 1015 LLINs, 678(66.8\%) were used.

Majority $417(64.3 \%)$ of the households not tucked the net under the mattress and significant number of household $65(10 \%)$ had one or more LLINs with tears or holes. Twenty nine $(4.5 \%)$ of the household repaired the tears with some other materials.

In this study, the proportion of children under 5 years age who slept under the net during early morning survey was $326(71.64 \%)$, 83(69\%) for pregnant mother and 1,152(68\%) other population group (Table 2 ).

The major reasons indicated by $204(31.4 \%$ ) of the households who didn't utilized the LLINs were; forgetfulness among 94(46.6\%) and considering the LLINs is too hot $32(15.7 \%)$ (Figure1).

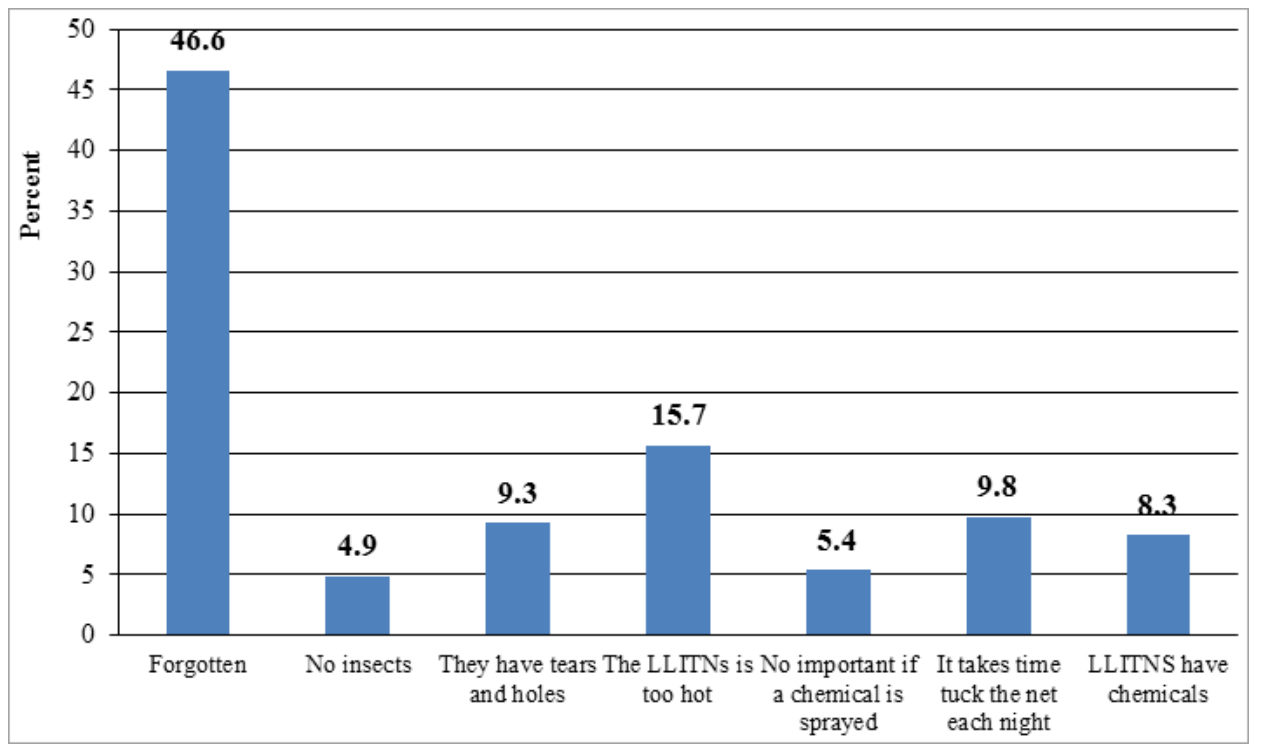

Figure 1. Reasons for not utilizing LLINs among households in Raya-Alamata district, Tigray, 2013.

\subsection{Factors Affecting Long Lasting Treated Nets Utilization}

In order to determine what factors influence the utilization of LLINs, several socio demographic and malaria related factors were entered in stepwise regression. As can be seen on marital status, occupational status and number of long lasting insecticidal nets was found to be significantly associated with the utilization. Other variables like socio demographic (age, gender, educational status) did not show any statistical significance.

Head of households whose marital status divorced were $56.9 \%$ [AOR $(95 \% \mathrm{CI})=0.431(0.186-0.997)]$ less likely to use net compared to those head of households whose marital status were married. Household head who were farmer were $70.3 \%$ [AOR $(95 \% \mathrm{CI})=0.297(0.113-0.781)]$ less likely to use net compared to those Government /Private Employed head. Households ownership for at least one mosquito net and use of the nets were $91.5 \%$ [AOR $(95 \% \mathrm{CI})=0.085$ (0.26-0.281)] less likely to use bed nets compared to those who have three bed nets in their home. Households ownership for at least two mosquito net and use of the nets were $77.7 \%$ [AOR $(95 \% \mathrm{CI})=0.223(0.26-0.281)]$ less likely to use bed nets compared to those who have three bed nets in their home (Table 3).

Table 3. Factors affecting utilization of long lasting treated nets among households in Raya-Alamata district, Tigray,Ethiopia, 2013.

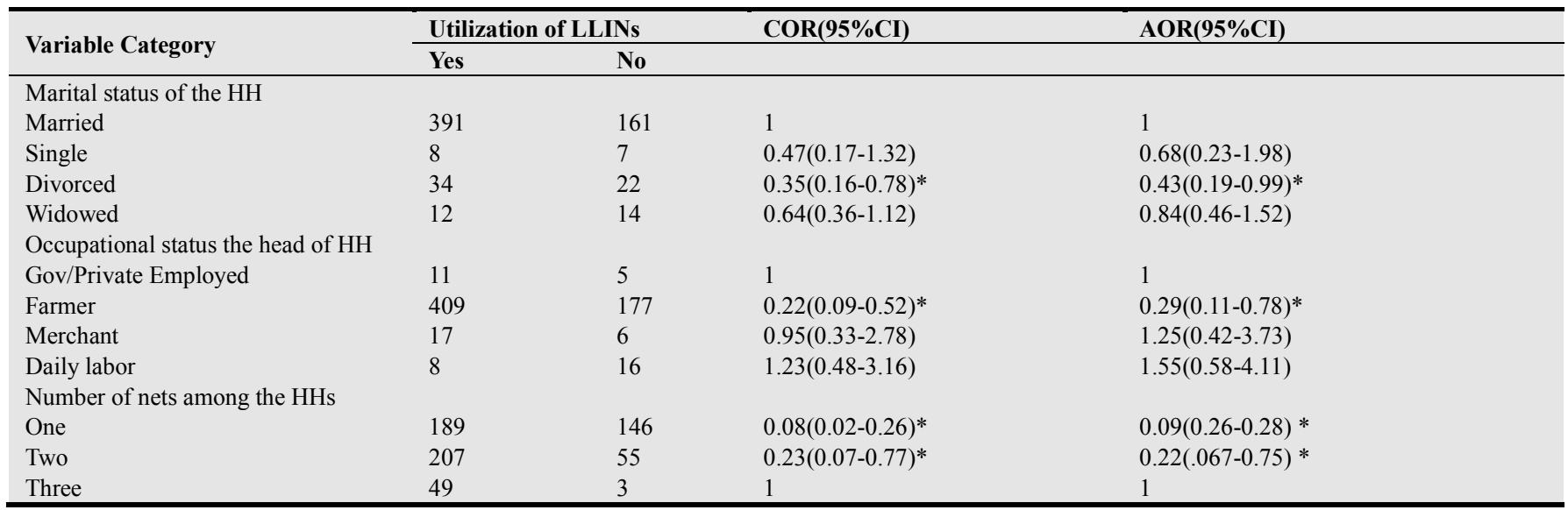

*Significant at $\mathrm{P}<0.05$ 


\section{Discussion}

Long Lasting Insecticide Treated Nets have become important tools that provide a simple and effective means of preventing malaria in highly endemic areas like RayaAlamata district. Though, the Ethiopian Federal Ministry of Health works substantially to increase ownership of LLINs in each malarious area of the households, barriers has been indicated in terms of utilization. So this study tries to assess the utilization of LLINs and factors affecting the utilization in the study area.

In this study the utilization of LLINs is $68.6 \%$ and significant number, $31.4 \%$ of households do not use their net. These findings justify that there is a considerable discrepancy between the distribution and utilization of the net. In addition, the finding indicates that even though the household own the net they do not utilize for the intended purpose and this may affect the program intervention. Comparing this finding with a study done in Arbaminch Town, Ethiopia that, $73 \%$ of the household utilized the net the night preceding the study, the current finding is slightly lower. This discrepancy might be due to the fact that the current study was conducted among the rural households of Ethiopia (7). This study is based on the actual early morning observation. The percentage of utilization even lower than the above result because, a significant number $(64.3 \%)$ of households not tucked the net under the mattress and some $(10 \%)$ of the household's LLINs have tears or holes this failed to protect man-vector contact and malaria infection.

Malaria mostly affects pregnant women and children and LLINs are beneficial in reducing the burden of malaria in this group. Levels of perceived advantages of sleeping under the net by these groups are extremely high (11). In this study the proportion of under 5 children and pregnant women who slept under the LLINs during early morning survey was $71.64 \%$ and $69 \%$ respectively. This finding is high than a study done in Arbaminch Town, Ethiopia which is $35 \%$ of pregnant women and $40.3 \%$ children slept under the net. This difference may due to the fact that in rural setting there is appropriate health information regarding the use of LLINs during the provision of the nets. The presence of health extension workers and women development army plays a great role in prevention of malaria in these vulnerable groups (7). When interpreted with reference to the Abuja targets in which $60 \%$ of under-fives and pregnant women are expected to sleep under ITNs by the year 2005 (6) and the national stands on achieving the figures set on the Abuja Declaration by 2007 (12) The use of LLINs by these specific groups in the current finding is higher than the standard also.

Multivariate logistic regression analysis suggested that the number of nets among the HHs is one of the predictor variables for household's utilization of LLINs. This study explored that household's ownership and use of the net for at least one LLITN and for two LLINs were $92.1 \%$ and $77 \%$ less likely to use the net comparing with households having three LLINs in their home respectively. This finding is similar to the study done in Haragea Gursum, Ethiopia that household's ownership for at least one mosquito net was $37.6 \%$ less likely to use bed nets than those who have more bed nets (13). Similarly a study done in Butajira area south central Ethiopia indicated that, presence of two and more net owned in the household were significantly associated with increased household utilization of the net (14).

This study revealed that households whose heads were engaged as farmer were $70.3 \%$ less likely to use LLINs compared to those governmental and private workers. This might be due to the fact that governmental and private workers have more awareness than farmers because of their educational status or access to information. This finding was similar but higher in percent to a study done in Harargae, Ethiopia which indicates that households whose heads engaged as a farmer were $13.7 \%$ less likely to use bed net (20). In contrary, another study reviled that households with government employees were 13\% less likely to use bed nets and in the same way a study done in Butajira area reveled that farming occupation showed statistically significant association with highly likely to use LLINs $(11,14)$. This dissimilarity may be due to the socio demographic difference.

This study also showed that marital status was significantly associated with bed net utilization. Head of households whose marital status divorced were $56.9 \%$ less likely to use bed net compared to those head households whose marital statuses were married. This may be due the fact that either of married couples may have access to information and they may share it to prevent from disease.

When interpreting the findings of this study some points to be considering are the study was conducted in the second pick malaria transmission period in the district which is from May to April and many people feel they need to use the nets in this season. This finding indicates the maximum utilization of the LLINs and the utilization might be different in other seasons. The study tried to minimize respondent bias during the interview by direct observing of the actual utilization through early morning survey.

\section{Conclusion and Recommendation}

The findings of this study shows, the utilization of LLINs in the study area is low and there is a considerable discrepancy between the distribution by the government and utilization of the net. Availability of number of nets among the households, occupational status the head of households and marital status of the household are the predictor variables for household's utilization of LLINs.

Thus, to increase utilization of LLINs in the study community; target specific interventions at children under five years and pregnant women through services supported with local training and supervision so as to increase the utilization of LLINs per household and the proportion of vulnerable who are sleeping under bed net. Effective Information Education and Communication, IEC should be promoted to improve and sustain LLINs program through 
demonstration in health facilities, schools, farmer training center and other relevant places, adaptation of pictures to culturally acceptable aspect. The government should sustain and strengthen women health development army which promote maternal and child health care with regard to malaria and other disease prevention and control mechanism.

\section{Author Contribution}

GDA: Involved in proposal writing, designing, and recruitment and training of supervisors and data collectors, analysis and in all stage of the study. HLR: Involved in proposal writing, designing, analysis and write up of the study. AGT: Involved in proposal writing, designing, analysis and write-up and manuscript preparation of the study. Finally all authors have been read and approved the manuscript.

\section{Acknowledgements}

We are very indebted to forward our appreciation for Tigray Regional Health Bureau and Raya -Alamata District health and administration offices for their cooperation during the entire study. Most, we would also like to extend our gratitude to all study participants for their cooperation and commitment in responding to our interviews and to the data collectors and supervisors for their extensive devotion at fieldwork.

\section{References}

[1] World Health Organization. World Malaria Report 2010. Available:

http://www.Who.Int/Malaria/World_Malaria_Report_2010/En Index.Html

[2] World Health Organization. Malaria Report 2013. Available from: http://www.WHO.Int/Malaria/World_Malaria_Report_2013.

[3] Sebastian MS, Lemma H. Efficiency of the health extension programme in Tigray, Ethiopia: a data envelopment analysis. H.BMC Int Health Hum Rights. 2010.
[4] Paulander J, Olsson H, Lemma $\mathrm{H}$ etal. Knowledge, attitudes and practice about malaria in rural Tigray, Ethiopia. Glob Health Action. 2009:2.

[5] Lemma H, Byass P, Desta A, etal. Deploying artemetherlumefantrine with rapid testing in Ethiopian communities: impact on malaria morbidity, mortality and healthcare resources. Trop Med Int Health. 2010.

[6] Roll Back Malaria. Global Strategic Plan 2005-2015. Geneva: Roll Back Malaria Partnership; 2008:52.

[7] Ayalew A. Amsalu F. Utilization Of Insecticide Treated Nets In Arbaminch Town And The Malarious Villages of Arbaminch Zuria District, Southern Ethiopia. Ethiop.J.Health Dev. 2009: 23(3).

[8] Unpublished Rural Alamata woreda annual report 2004.

[9] Federal Democratic Republic of Ethiopia, Ministry of Health. National Strategic Plan for Malaria Prevention Control and Elimination in Ethiopia. 2010 - 2015. March 2009; Addis Ababa.

[10] Zewdneh, T.Tadesse, D and Dawit K. Knowledge, Attitude and Practice (KAP) about Insecticide Treated Net (ITN) usage against Malaria in Kolla Tembien district, Tigray, Ethiopia. 2011: 3(2):64-77.

[11] Berhane H, Ahmed A. Assessment Of Insecticide Treated Nets Coverage For Malaria Control In Kafta-Humera District, Tigray. Possession vs. Use by High-Risk Groups. Ethiop.J.Health Dev. 2008; 22(3):259-267.

[12] Federal Democratic Republic of Ethiopia, Ministry of Health. Insecticide Treated Nets (ITNs): National Strategic Plan for Going to Scale with Coverage and Utilization in Ethiopia, 2004-2007. August 2004.

[13] Sibhatu et al. Determinants of ownership and utilization of insecticide treated bed nets for malaria control in eastern Ethiopia. 2012.

[14] Adugna W, Wakgari D, Ahmed A, Bernt L. Ownership and use of long-lasting insecticidal nets for malaria prevention in Butajira area, south-central Ethiopia: complex samples data analysis. BMC Public Health 2014, 14:99 doi: 10.1186/14712458-14-99. 